\title{
The Pierre Auger Observatory scaler mode for the study of solar activity modulation of galactic cosmic rays
}

\section{The Pierre Auger collaboration}

The Pierre Auger Observatory,

Malargüe, Argentina

ABSTRACT: Since data-taking began in January 2004, the Pierre Auger Observatory has been recording the count rates of low energy secondary cosmic ray particles for the self-calibration of the ground detectors of its surface detector array. After correcting for atmospheric effects, modulations of galactic cosmic rays due to solar activity and transient events are observed. Temporal variations related with the activity of the heliosphere can be determined with high accuracy due to the high total count rates. In this study, the available data are presented together with an analysis focused on the observation of Forbush decreases, where a strong correlation with neutron monitor data is found.

KEYWORDS: Cherenkov detectors; Large detector systems for particle and astroparticle physics; Particle detectors 


\section{Contents}

1 Introduction $\quad 1$

2 The scaler mode $\quad 3$

3 Scaler data treatment $\quad 3$

4 Data interpretation $\quad 4$

$\begin{array}{llr}5 & \text { Conclusions } & 8\end{array}$

\section{Introduction}

The heliospheric modulation of Galactic Cosmic Rays (GCRs) observed near Earth is controlled by the solar activity. In particular it is influenced by the variability of solar wind (SW) conditions, due to the solar cycle modulation (e.g., through changes of the global structure and polarity of the magnetic field in the heliosphere) and to transient eruptions of solar ejecta. The variation of the diffusion coefficient due to different levels and properties of interplanetary magnetic field (IMF) fluctuations can also affect this modulation.

The observed modulation of cosmic rays (CRs) intensity provides information about the transport of particles in the inner and outer heliosphere, and also about particles coming into the solar system from the Local Interstellar medium, see for example [1]. In particular GCRs intensity observed near Earth have shown a long-term modulation (associated with the solar cycle) and short-term variations, such as Forbush decreases [2]. These transient events are short-term periods of strong depressions of the cosmic ray flux observed at Earth. As observed in neutron monitors (NMs) and muon detectors, the structure of Forbush decreases is asymmetrical, starting suddenly and recovering smoothly with typical recovery times of the order of several days. A typical Forbush decrease is caused by the transit of a solar ejecta (i.e., the interplanetary manifestation of a Coronal Mass Ejection, CME) near Earth [3].

Dedicated neutron monitors are being used since several decades ago as ground observatories to measure variations of CRs intensities. More recently many ground based CR detectors aiming at $\mathrm{CR}$ physics at higher energies are also being used for these studies.

The Pierre Auger Observatory [4] was designed to study the physics of cosmic rays at the highest energies. It is located in Malargüe, Argentina ( $69.3^{\circ} \mathrm{W}, 35.3^{\circ} \mathrm{S}, 1400 \mathrm{~m}$ a.s.l.). Detection of cosmic rays of energies above $10^{18} \mathrm{eV}$ requires the instrumentation of a huge detection area, due to the extremely low flux of particles at these extreme energies. The Auger Observatory combines two detection techniques in a hybrid design: the observation of the fluorescence light produced by the secondary particles as they propagate through the atmosphere, and the direct measurement of particles reaching ground level. The layout of the Pierre Auger Observatory is shown in figure 1. 


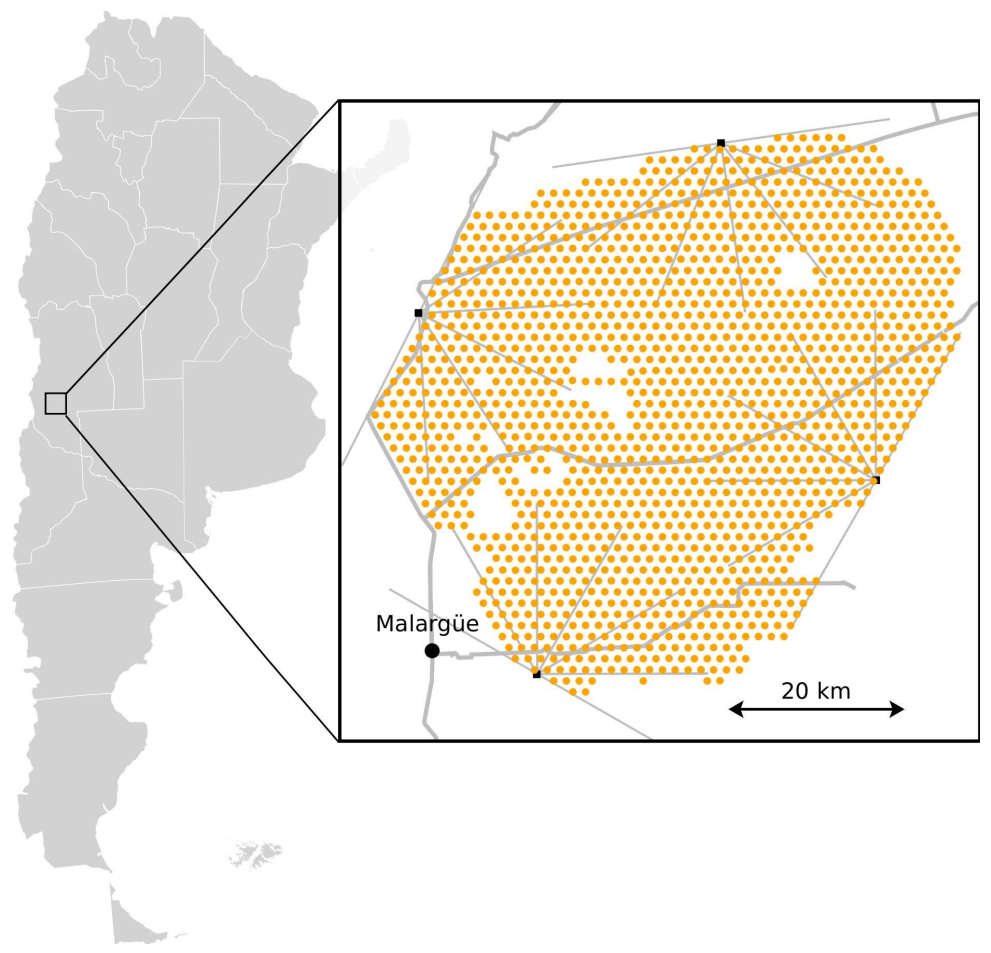

Figure 1. Layout of the Pierre Auger Observatory at Malargüe, Argentina. The position of the four FD buildings surrounding the SD array are indicated by the black squares, with gray lines indicating the field of view of the six telescopes at each building. Orange dots mark the position of each of the 1600 waterCherenkov detectors, with a $1.5 \mathrm{~km}$ spacing between nearest neighbours.

The interaction of a high-energy particle with the atmosphere produces a cascade with a huge number of secondary particles called an Extensive Atmospheric Shower (EAS). The longitudinal development of the EAS is followed by the Fluorescence Detector [5] (FD) consisting of 24 telescopes grouped in units of six telescopes at four different locations (Los Leones, Coihueco, Loma Amarilla and Los Morados). FD operates only in moonless and clear-weather nights, with an operational average duty cycle of $13 \%$ of the total time. It overlooks the atmosphere above a $3000 \mathrm{~km}^{2}$ area, where the Surface Detector (SD) [4] is deployed.

The SD is an array of more than 1600 water-Cherenkov detectors placed in a triangular grid with a spacing of $1500 \mathrm{~m}$. The lateral (transverse) distribution of secondary particles of EAS at ground level is sampled by using the SD array. Each water-Cherenkov detector consists of a polyethylene tank $\left(10 \mathrm{~m}^{2}\right.$ area) containing 12 tonnes of high-purity water in a highly-reflective Tyvek $^{\circledR}$ liner bag [6], providing a total of about $16000 \mathrm{~m}^{2}$ of collection area for the full SD array. Cherenkov radiation generated by the passage of charged particles through the water volume in each detector is collected by three 9" Photonis XP1805PA/1 photomultiplier tubes (PMTs). The output signals from the PMTs (one directly from the anode and the other one from the last dynode with an amplification factor of 32 to increase the dynamic range) are processed by six 10-bit flash analog-to-digital converters (FADC) with a sampling rate of $40 \mathrm{MHz}$ (25 ns per time bin). A GPS system is used for timing and synchronisation. Each detector is powered by two solar panels and batteries, working as an autonomous station linked to the central data acquisition system (CDAS) 
in Malargüe through a dedicated radio network. Typically, more than $98 \%$ of the detectors are operational at any given time.

The calibration is performed by local electronics in each detector [7], given the limited available bandwidth (1200 bps per station [8]). The signal produced in a detector by a vertical and central through-going muon, known as the vertical-equivalent muon (VEM), is used as a reference for calibration. Signals recorded by the detectors are converted to units of VEM.

The detectors are sensitive to charged particles in the EAS at ground level (essentially dominated by $\mu^{ \pm}$and $e^{ \pm}$), as well as to high energy photons, as these convert in $e^{+} e^{-}$pairs in the water volume. While the detector acts as a calorimeter for $\gamma$ and $e^{ \pm}$, the energy deposited by energetic muons (with energies $E \gtrsim 350 \mathrm{MeV}$ ) is nearly proportional to the track length of the muon through the detector, as the water volume is not enough to stop them. These muons therefore produce a characteristic hump in a background charge histogram $[9,10]$ that can be used for calibration [7]. Using a test detector with an external trigger from a muon hodoscope, the muon hump in the charge histogram was found to be at $(1.03 \pm 0.02) \operatorname{VEM}[10,11]$.

Given the typical energy losses of muons in water for high energy muons [12], $(d E / d x)_{\mu} \simeq$ $2 \mathrm{MeV} \mathrm{cm}{ }^{-1}$, a vertical and central muon deposits $\sim 240 \mathrm{MeV}$ in a detector with $1.2 \mathrm{~m}$ depth of water. At the operating gain of the SD detector, the correspondence between the amplitude of the pulse in FADC counts and the energy deposited by a particle in the detector is $1 \mathrm{FADC} \simeq 5 \mathrm{MeV}$. For details, see [7].

\section{The scaler mode}

The single particle technique [13], used in many cosmic rays experiments, consists in recording low threshold rates (scalers) with all the surface detectors of the array. Transient events such as Gamma Ray Bursts (GRBs) [14-17] and solar flares [18, 19] are expected to be seen as a significant change of the counting rates from the expected value.

In March 2005, a first set of scalers was implemented in all the detectors of the SD [14], for the search of GRBs and for long term stability and monitoring studies. These counters registered any signal above a threshold of 3 FADC counts, corresponding to an energy of $\sim 15 \mathrm{MeV}$ deposited by particles that reach the detector in a $25 \mathrm{~ns}$ bin. The typical average scaler rates obtained were around 3800 counts per second per detector, i.e, 380 counts s $\mathrm{s}^{-1} \mathrm{~m}^{-2}$. A new scaler mode, with a threshold of 3 FADC counts and an upper bound of 20 FADC counts $(\sim 100 \mathrm{MeV})$ was introduced in September 2005 to remove signals produced by muons and thus to reduce the background noise for GRBs searches, reducing the average scaler rate of each detector to $\sim 2000$ counts per second [14]. The rates at each detector are registered for every second and sent to the CDAS for storage and further analysis. The main characteristics of the scalers in both periods are summarised in table 1 .

\section{Scaler data treatment}

Scaler rates are influenced by the intrinsically non-constant rate of low energy-particles, atmospheric conditions and eventual instrumental instabilities. These effects have to be taken into account before searching for transient events (that last longer than a few minutes) and for studying the long term solar modulation of the galactic cosmic ray flux [19]. 
Table 1. Main characteristics of count rates for Auger scalers in both periods as defined in section 2. The collecting area range is due to the progressive development of the array to its completion in 2008.

\begin{tabular}{|l|c|c|c|}
\hline Period & $\begin{array}{c}\text { Energy range } \\
{[\mathrm{MeV}]}\end{array}$ & $\begin{array}{c}\text { Average scaler rate } \\
{\left[\text { counts s }{ }^{-1} \mathrm{~m}^{-2}\right]}\end{array}$ & $\begin{array}{c}\text { Collection area } \\
{\left[\mathrm{m}^{2}\right]}\end{array}$ \\
\hline I: 01 Mar 2005 - 20 Sep 2005 & $E \gtrsim 15$ & $\sim 380$ & $6660-8420$ \\
\hline II: After 20 Sep 2005 & $15 \lesssim E \lesssim 100$ & $\sim 200$ & $8420-16250$ \\
\hline
\end{tabular}

At the very low threshold of 3 FADC counts used for the scaler mode, some detectors show instabilities evidenced at the level of a few FADC counts. These do not affect the normal operation mode of the SD, which uses thresholds of about 170 FADC counts for detection of EAS. Extra care is therefore required to select the detectors to be used in the scaler analysis. Stations with rates lower than 500 counts $\mathrm{s}^{-1}$ are removed, as such a low rate is an indication of temporary instability. For each individual second we also discard data from those stations with extreme counting rates (upper and lower 2.5\%). The second step is to remove those periods where less than $97 \%$ of the array is in operation, resulting in a total loss of less than $10 \%$ of data. This step is needed because individual stations have different average counting rates, due to different reasons, such as detector calibration or pressure effects caused by the different altitudes at which detectors are deployed.

Data for the two different periods was analysed independently. For both periods the average scaler rate was computed for each station over the lifetime of the detector. Detectors showing an RMS of more than twice the square root of that rate were excluded, keeping more than $90 \%$ of the stations after this cut. Brief spurious events (such as high frequency noise produced by lightning) were removed by computing the average scaler rate for each detector over a 5 minutes period in which data is available for at least 4 minutes.

It is worthwhile to note that given the huge counting rate $\left(>3 \times 10^{6}\right.$ counts s$^{-1}$ for the full SD array, $\sim 10^{9}$ particles counted every 5 minutes), the statistical accuracy is well below $1 \%$ and the measurement precision is dominated by possible systematics.

Atmospheric pressure variations are known to modify the flux of secondary particles at ground level, due to the variation of mass of atmosphere above the detector: an increase in the atmospheric pressure is correlated with a reduction in the background rate. The average scaler rate for the first ten days of May 2007 is shown in figure 2, compared with the atmospheric pressure, as measured by one of several weather stations operated at the observatory. The correlation corresponds to about $-2.7 \pm 0.2$ (stat.) \%o per hPa for Period I and $-3.6 \pm 0.2$ (stat.) \%o per hPa for Period II. Other atmospheric effects such as those discussed in [20] were studied but their correlation with scaler rate is small (less than 1 part in $10^{4}$ per degree Celsius for the temperature, for example), in particular when compared to the daily modulation discussed in section 4 .

\section{Data interpretation}

The daily variation of the averaged scaler rate is shown in figure 3. After atmospheric pressure correction, a $0.25 \%$ modulation remains, with a maximum at $17 \mathrm{~h} 45 \mathrm{UT}$ ( $14 \mathrm{~h} 45$ local time). While its phase and amplitude are comparable with the expected solar modulation [21], other atmospheric (air density, temperature) and instrumental systematics cannot be ruled out. 


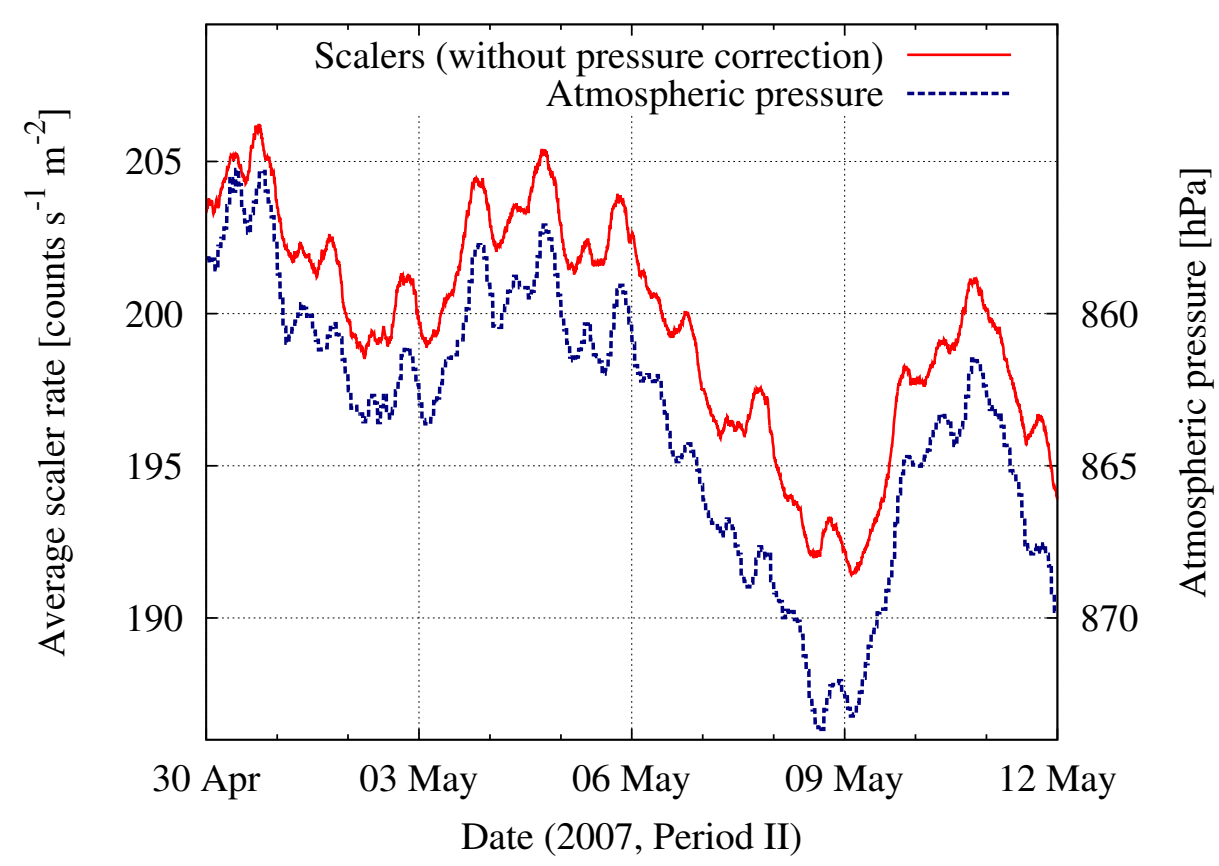

Figure 2. Scaler rate averaged over time bins of 5 minutes and over all detectors in the array (solid line); and atmospheric pressure (dashed line, note the reversed scale for pressure) for the first 10 days of May 2007.

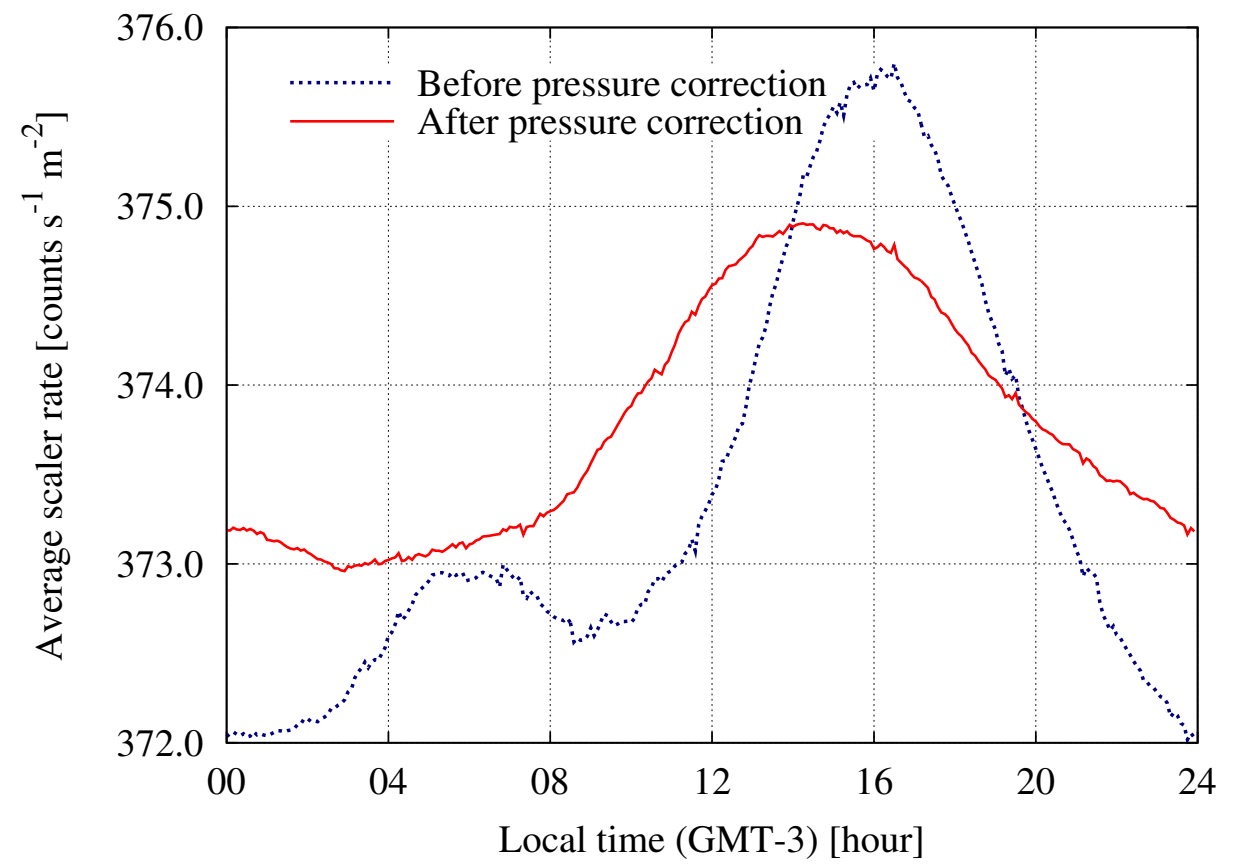

Figure 3. Average scaler rates for period I as a function of local hour of day (ARS, GMT-3) for the original data (dotted line) and for the atmospheric pressure corrected data (solid line). 

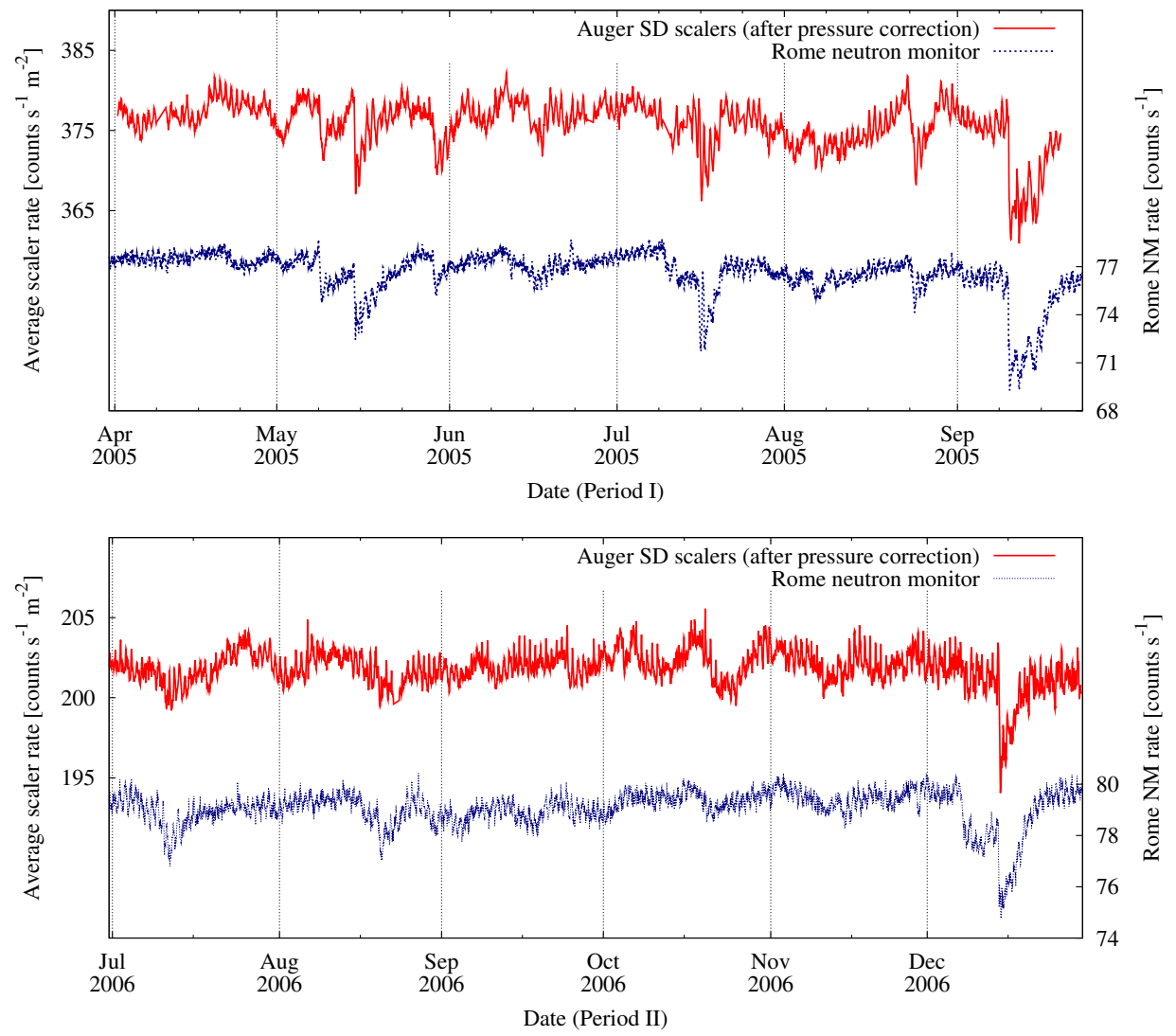

Figure 4. Pierre Auger Observatory scaler rates (red solid lines) after atmospheric pressure correction, compared to Rome neutron monitor rate (blue dotted line), for both periods, before (top, Period I full data set) and after 20 September 2005 (bottom, from 01 Jul 2006 to 31 Dec 2006). Note that scales are different for each observatory.

To show that the pressure corrected data set is suitable for the study of solar activity, the SD scaler rates are compared with data from Rome neutron monitor $[22]\left(41.9^{\circ} \mathrm{N}, 12.5^{\circ} \mathrm{E}, 6.3 \mathrm{GV}\right.$ rigidity cut-off). The agreement found is shown in figure 4: Forbush decreases are clearly visible in the scaler data for both periods. The upper threshold introduced in September 2005, intended to optimise signal over noise ratio for GRB detection, is not optimised for these studies, where the muon flux at ground level might be better correlated to the primary cosmic ray flux than the electron one. A study including different thresholds is underway.

As an example, the evolution of the scaler rates in Malargüe $\left(35.3^{\circ} \mathrm{S}, 69.3^{\circ} \mathrm{W}, 9.5 \mathrm{GV}\right.$ rigidity cut-off [23]) during the 15 May 2005 Forbush event is shown in figure 5. When compared with the May 2005 reference rate of 377 counts s $\mathrm{m}^{-1}$, a $(-2.9) \%$ variation is observed, peaked at 15 May 2005 08:05 UTC, taking $\sim 6 \mathrm{~h} 45$ to reach the peak value. After the rapid reduction in the flux of secondary particles, a recovery time of about 9 days is observed. By fitting an exponential function for the recovery (red dot-dashed line), $R(t)=R_{0}-a \exp (-t / \tau)$, a baseline $R_{0}=377.7 \pm$ 0.1 counts s $\mathrm{s}^{-1} \mathrm{~m}^{-2}$ (red dotted line) and a time constant of $\tau=2.21 \pm 0.18$ (stat) days are obtained. 


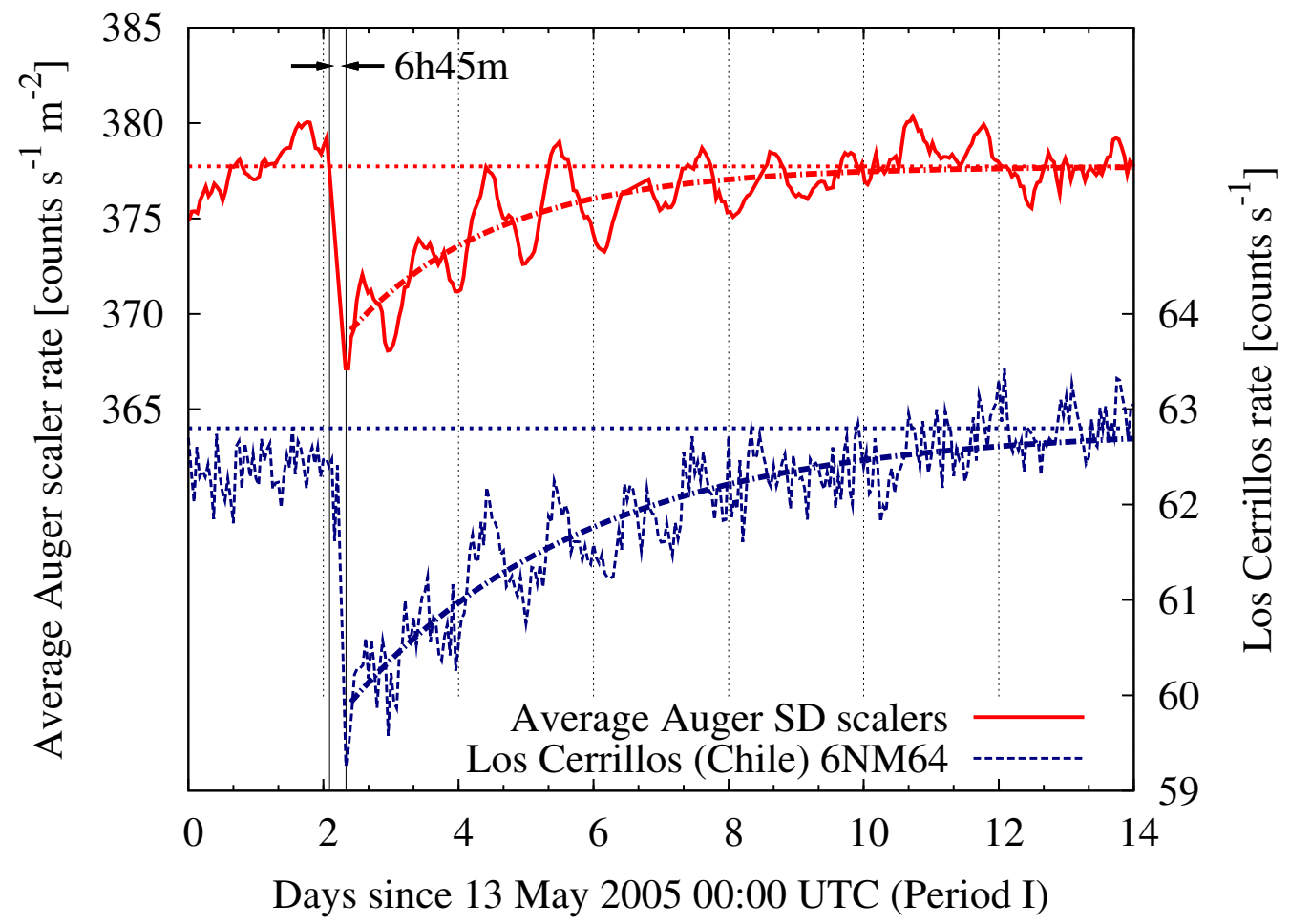

Figure 5. Pressure-corrected scaler rate (red solid line) for the 15 May 2005 Forbush event, compared with the Los Cerrillos (Chile) neutron monitor rate (blue dashed line). A 2.9\% decrease is observed, peaked at 15 May 2005 08:05 UTC, taking about 6h45 (olive dotted vertical lines) to reach the peak value, and about nine days to recover up to the scaler rate average for May 2005 (red dashed line). An exponential function has been fitted on both rates (red and blue dot-dashed lines) as described in the text. Similar daily variations in the flux are seen at both observatories.

A comparison with observations from the close-by Los Cerrillos Observatory 6NM64 neutron monitor (Chile) [24] $\left(33.3^{\circ} \mathrm{S}, 70.4^{\circ} \mathrm{W}, 10.8 \mathrm{GV}\right.$ rigidity cut-off) is also shown in figure 5 (blue dashed line). Since the Forbush event of 15 May occurred during the recovery phase of a previous Forbush decrease, which started on 08 May 2005, the baseline for Los Cerrillos rate increases suddenly at the end of May 2005, and the determination of the baseline by leaving it as a free parameter in the fit is not possible. Therefore, the baseline of Los Cerrillos was determined from the neutron monitor rates of the first six days of May 2005, combined with the recovery phase of the studied Forbush decrease, obtaining $R_{0}=62.8 \pm 1$ counts s$^{-1}$ (blue dotted line). A $4.8 \%$ reduction in the neutron rate is observed, with an exponential recovery (blue dot-dashed line) with time constant $\tau=3.52 \pm 0.12$ (stat) days. An agreement between the neutron monitor and the scaler data is found in the determination of the starting point and the duration of the onset (vertical dashed lines in figure 5). Since both observatories have a similar rigidity cut-off, the difference in the observed time constants reflects the higher energy threshold of the Auger SD scalers with respect to the neutron monitor. 


\section{Conclusions}

Observations of CR intensities play an important role in our understanding of the interplanetary and interstellar media, and also on our knowledge on physical mechanisms involving the interaction between charged particles and the magnetized plasma in the heliosphere. Some recent studies have started to combine ground observations of CRs with observations of energetic particles made by spacecrafts, with very promising results [25-27].

We have described how low energy radiation rates are registered with high statistics at all the surface detector array stations of the Pierre Auger Observatory, with a current collection area of more than $16000 \mathrm{~m}^{2}$. A data processing method, based on a previous one intended for the search of GRBs, has been implemented to observe modulation effects. After correcting for pressure, the behaviour of scaler rates shows a good agreement with data from neutron monitors. WaterCherenkov detectors, operating in scaler mode, are therefore highly sensitive to Forbush decreases and other transient events related to solar activity modulation of galactic cosmic rays.

Additional analyses of scaler rates at individual detectors, different scaler lower and upper thresholds, and simulations of low energy cosmic rays (to determine the primary energies responsible for the observed modulation) are underway.

\section{Acknowledgments}

The successful installation and commissioning of the Pierre Auger Observatory would not have been possible without the strong commitment and effort from the technical and administrative staff in Malargüe.

We are grateful to Prof. Enrique G. Cordaro and the Observatorio Radiación Cósmica of the Universidad de Chile, who kindly provided the neutron monitor data of Los Cerrillos Observatory.

We are very grateful to the following agencies and organizations for financial support: Comisión Nacional de Energía Atómica, Fundación Antorchas, Gobierno De La Provincia de Mendoza, Municipalidad de Malargüe, NDM Holdings and Valle Las Leñas, in gratitude for their continuing cooperation over land access, Argentina; the Australian Research Council; Conselho Nacional de Desenvolvimento Científico e Tecnológico (CNPq), Financiadora de Estudos e Projetos (FINEP), Fundação de Amparo à Pesquisa do Estado de Rio de Janeiro (FAPERJ), Fundação de Amparo à Pesquisa do Estado de São Paulo (FAPESP), Ministério de Ciência e Tecnologia (MCT), Brazil; AVCR AV0Z10100502 and AV0Z10100522, GAAV KJB300100801 and KJB100100904, MSMT-CR LA08016, LC527, 1M06002, and MSM0021620859, Czech Republic; Centre de Calcul IN2P3/CNRS, Centre National de la Recherche Scientifique (CNRS), Conseil Régional Ile-de-France, Département Physique Nucléaire et Corpusculaire (PNC-IN2P3/CNRS), Département Sciences de l'Univers (SDU-INSU/CNRS), France; Bundesministerium für Bildung und Forschung (BMBF), Deutsche Forschungsgemeinschaft (DFG), Finanzministerium BadenWürttemberg, Helmholtz-Gemeinschaft Deutscher Forschungszentren (HGF), Ministerium für Wissenschaft und Forschung, Nordrhein-Westfalen, Ministerium für Wissenschaft, Forschung und Kunst, Baden-Württemberg, Germany; Istituto Nazionale di Fisica Nucleare (INFN), Istituto Nazionale di Astrofisica (INAF), Ministero dell'Istruzione, dell'Università e della Ricerca (MIUR), Italy; Consejo Nacional de Ciencia y Tecnología (CONACYT), Mexico; Ministerie van 
Onderwijs, Cultuur en Wetenschap, Nederlandse Organisatie voor Wetenschappelijk Onderzoek (NWO), Stichting voor Fundamenteel Onderzoek der Materie (FOM), Netherlands; Ministry of Science and Higher Education, Grant Nos. 1 P03 D 01430 and N N202 207238, Poland; Fundação para a Ciência e a Tecnologia, Portugal; Ministry for Higher Education, Science, and Technology, Slovenian Research Agency, Slovenia; Comunidad de Madrid, Consejería de Educación de la Comunidad de Castilla La Mancha, FEDER funds, Ministerio de Ciencia e Innovación and Consolider-Ingenio 2010 (CPAN), Generalitat Valenciana, Junta de Andalucía, Xunta de Galicia, Spain; Science and Technology Facilities Council, United Kingdom; Department of Energy, Contract Nos. DE-AC02-07CH11359, DE-FR02-04ER41300, National Science Foundation, Grant No. 0450696, The Grainger Foundation USA; ALFA-EC / HELEN, European Union 6th Framework Program, Grant No. MEIF-CT-2005-025057, European Union 7th Framework Program, Grant No. PIEF-GA-2008-220240, and UNESCO.

\section{The Pierre Auger collaboration}

P. Abreu ${ }^{73}$, M. Aglietta ${ }^{55}$, E.J. Ahn ${ }^{89}$, D. Allard ${ }^{31}$, I. Allekotte ${ }^{1}$, J. Allen ${ }^{92}$, J. Alvarez Castillo $^{66}$, J. Alvarez-Muñiz ${ }^{80}$, M. Ambrosio ${ }^{48}$, A. Aminaei ${ }^{67}$, L. Anchordoqui ${ }^{106}$, S. Andringa ${ }^{73}$, T. Antičić ${ }^{25}$, A. Anzalone ${ }^{54}$, C. Aramo ${ }^{48}$, E. Arganda ${ }^{77}$, K. Arisaka ${ }^{97}$, F. Arqueros ${ }^{77}$, H. Asorey ${ }^{1}$, P. Assis ${ }^{73}$, J. Aublin ${ }^{33}$, M. Ave ${ }^{37,98}$, M. Avenier ${ }^{34}$, G. Avila ${ }^{10}$, T. Bäcker ${ }^{43}$, D. Badagnani ${ }^{6}$, M. Balzer ${ }^{38}$, K.B. Barber ${ }^{11}$, A.F. Barbosa ${ }^{14}$, R. Bardenet ${ }^{32}$, S.L.C. Barroso ${ }^{20}$, B. Baughman ${ }^{94}$, J.J. Beatty ${ }^{94}$, B.R. Becker ${ }^{103}$, K.H. Becker ${ }^{36}$, A. Bellétoile ${ }^{34}$, J.A. Bellido ${ }^{11}$, S. BenZvi ${ }^{105}$, C. Berat ${ }^{34}$, T. Bergmann ${ }^{38}$, X. Bertou ${ }^{1}$, P.L. Biermann ${ }^{40}$, P. Billoir ${ }^{33}$, F. Blanco ${ }^{77}$, M. Blanco ${ }^{78}$, C. Bleve ${ }^{36,47}$, H. Blümer ${ }^{39,37}$, M. Boháčová ${ }^{98,27}$, D. Boncioli ${ }^{49}$, C. Bonifazi ${ }^{23,33}$, R. Bonino ${ }^{55}$, N. Borodai ${ }^{71}$, J. Brack ${ }^{87}$, P. Brogueira ${ }^{73}$, W.C. Brown ${ }^{88}$, R. Bruijn ${ }^{83}$, P. Buchholz ${ }^{43}$, A. Bueno ${ }^{79}$, R.E. Burton ${ }^{85}$, N.G. Busca ${ }^{31}$, K.S. Caballero-Mora ${ }^{39}$, L. Caramete $^{40}$, R. Caruso ${ }^{50}$, A. Castellina ${ }^{55}$, O. Catalano ${ }^{54}$, G. Cataldi ${ }^{47}$, L. Cazon $^{73}$, R. Cester ${ }^{51}$, J. Chauvin ${ }^{34}$, A. Chiavassa ${ }^{55}$, J.A. Chinellato ${ }^{18}$, A. Chou ${ }^{89,92}$, J. Chudoba ${ }^{27}$, R.W. Clay ${ }^{11}$, E. Colombo ${ }^{2}$, M.R. Coluccia ${ }^{47}$, R. Conceiçãa ${ }^{73}$, F. Contreras ${ }^{9}$, H. Cook ${ }^{83}$, M.J. Cooper ${ }^{11}$, J. Coppens ${ }^{67,69}$, A. Cordier ${ }^{32}$, U. Cotti ${ }^{65}$, S. Coutu ${ }^{95}$, C.E. Covault ${ }^{85}$, A. Creusot $^{75}$, A. Criss ${ }^{95}$, J. Cronin ${ }^{98}$, A. Curutiu ${ }^{40}$, S. DagoretCampagne $^{32}$, R. Dallier ${ }^{35}$, S. Dasso ${ }^{7,4}$, K. Daumiller ${ }^{37}$, B.R. Dawson ${ }^{11}$, R.M. de Almeida ${ }^{18,23}$, M. De Domenico ${ }^{50}$, C. De Donato ${ }^{66,46}$, S.J. de Jong ${ }^{67}$, G. De La Vega ${ }^{8}$, W.J.M. de Mello Junior $^{18}$, J.R.T. de Mello Neto ${ }^{23}$, I. De Mitrii ${ }^{47}$, V. de Souza ${ }^{16}$, K.D. de Vries ${ }^{68}$, G. Decerprit ${ }^{31}$, L. del Peral ${ }^{78}$, O. Deligny ${ }^{30}$, A. Della Selva ${ }^{48}$, H. Dembinski ${ }^{37}$, A. Denkiewicz ${ }^{2}$, C. Di Giulio $^{49}$, J.C. Diaz ${ }^{91}$, M.L. Díaz Castro ${ }^{15}$, P.N. Diep ${ }^{107}$, C. Dobrigkeit ${ }^{18}$, J.C. D’Olivo ${ }^{66}$, P.N. Dong ${ }^{107,30}$, A. Dorofeev ${ }^{87}$, J.C. dos Anjos ${ }^{14}$, M.T. Dova ${ }^{6}$, D. D’Urso ${ }^{48}$, I. Dutan ${ }^{40}$, J. Ebr ${ }^{27}$, R. Engel ${ }^{37}$, M. Erdmann ${ }^{41}$, C.O. Escobar ${ }^{18}$, A. Etchegoyen ${ }^{2}$, P. Facal San Luis ${ }^{98}$, H. Falcke ${ }^{67,70}$, G. Farrar ${ }^{92}$, A.C. Fauth ${ }^{18}$, N. Fazzini ${ }^{89}$, A.P. Ferguson ${ }^{85}$, A. Ferrero ${ }^{2}$, B. Fick ${ }^{91}$, A. Filevich ${ }^{2}$, A. Filipčič ${ }^{74,75}$, I. Fleck ${ }^{43}$, S. Fliescher ${ }^{41}$, C.E. Fracchiolla ${ }^{87}$, E.D. Fraenkel ${ }^{68}$, U. Fröhlich ${ }^{43}$, B. Fuchs ${ }^{14}$, W. Fulgione ${ }^{55}$, R.F. Gamarra ${ }^{2}$, S. Gambetta ${ }^{44}$, B. García ${ }^{8}$, D. García Gámez ${ }^{79}$, D. Garcia-Pinto ${ }^{77}$, X. Garrido ${ }^{37}$, A. Gascon ${ }^{79}$, G. Gelmini ${ }^{97}$, H. Gemmeke ${ }^{38}$, K. Gesterling ${ }^{103}$, P.L. Ghia ${ }^{30,55}$, U. Giaccari ${ }^{47}$, M. Giller ${ }^{72}$, H. Glass ${ }^{89}$, M.S. Gold ${ }^{103}$, G. Golup ${ }^{1}$, F. Gomez Albarracin $^{6}$, M. Gómez Berisso ${ }^{1}$, P. Gonçalves ${ }^{73}$, D. Gonzalez ${ }^{39}$, J.G. Gonzalez ${ }^{39}$, B. Gookin ${ }^{87}$, D. Góra ${ }^{39,71}$, A. Gorgi ${ }^{55}$, P. Gouffon ${ }^{17}$, S.R. Gozzini ${ }^{83}$, E. Grashorn ${ }^{94}$, S. Grebe ${ }^{67}$, M. Grigat ${ }^{41}$, 
A.F. Grillo ${ }^{56}$, Y. Guardincerri ${ }^{4}$, F. Guarino ${ }^{48}$, G.P. Guedes ${ }^{19}$, J.D. Hague ${ }^{103}$, P. Hansen ${ }^{6}$, D. Harari ${ }^{1}$, S. Harmsma ${ }^{68,69}$, J.L. Harton ${ }^{87}$, A. Haungs ${ }^{37}$, T. Hebbeker ${ }^{41}$, D. Heck ${ }^{37}$, A.E. Herve ${ }^{11}$, C. Hojvat ${ }^{89}$, V.C. Holmes ${ }^{11}$, P. Homola ${ }^{71}$, J.R. Hörandel ${ }^{67}$, A. Horneffer ${ }^{67}$, M. Hrabovský ${ }^{28,27}$,

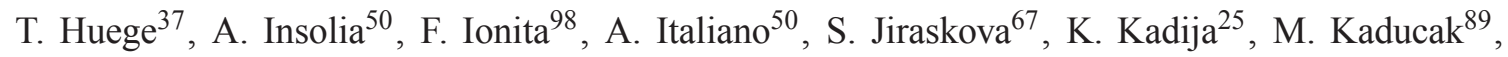
K.H. Kampert ${ }^{36}$, P. Karhan ${ }^{26}$, T. Karova ${ }^{27}$, P. $\operatorname{Kasper}^{89}$, B. Kégl ${ }^{32}$, B. Keilhauer ${ }^{37}$, A. Keivani ${ }^{90}$, J.L. Kelley ${ }^{67}$, E. Kemp ${ }^{18}$, R.M. Kieckhafer ${ }^{91}$, H.O. Klages ${ }^{37}$, M. Kleifges ${ }^{38}$, J. Kleinfeller ${ }^{37}$, J. Knapp ${ }^{83}$, D.-H. Koang ${ }^{34}$, K. Kotera ${ }^{98}$, N. Krohm ${ }^{36}$, O. Krömer ${ }^{38}$, D. Kruppke-Hansen ${ }^{36}$, F. Kuehn ${ }^{89}$, D. Kuempel ${ }^{36}$, J.K. Kulbartz ${ }^{42}$, N. Kunka ${ }^{38}$, G. La Rosa ${ }^{54}$, C. Lachaud ${ }^{31}$, P. Lautridou ${ }^{35}$, M.S.A.B. Leão ${ }^{22}$, D. Lebrun ${ }^{34}$, P. Lebrun ${ }^{89}$, M.A. Leigui de Oliveira $^{22}$, A. Lemiere ${ }^{30}$, A. Letessier-Selvon ${ }^{33}$, I. Lhenry-Yvon ${ }^{30}$, K. Link ${ }^{39}$, R. López ${ }^{61}$, A. Lopez Agüera $^{80}$, K. Louedec ${ }^{32}$, J. Lozano Bahilo ${ }^{79}$, A. Lucero ${ }^{2,55}$, M. Ludwig ${ }^{39}$, H. Lyberis ${ }^{30}$, M.C. Maccarone ${ }^{54}$, C. Macolino ${ }^{33}$, S. Maldera ${ }^{55}$, D. Mandat $^{27}$, P. Mantsch $^{89}$, A.G. Mariazzi ${ }^{6}$, V. Marin ${ }^{35}$, I.C. Maris ${ }^{33}$, H.R. Marquez Falcon ${ }^{65}$, G. Marsella ${ }^{52}$, D. Martello ${ }^{47}$, L. Martin ${ }^{35}$, O. Martínez Bravo ${ }^{61}$, H.J. Mathes ${ }^{37}$, J. Matthews ${ }^{90,96}$, J.A.J. Matthews ${ }^{103}$, G. Matthiae ${ }^{49}$, D. Maurizio ${ }^{51}$, P.O. Mazur ${ }^{89}$, G. Medina-Tanco ${ }^{66}$, M. Melissas ${ }^{39}$, D. Melo ${ }^{51}$, E. Menichetti ${ }^{51}$, A. Menshikov ${ }^{38}$, C. Meurer ${ }^{41}$, S. Mičanović25, M.I. Micheletti ${ }^{2}$, W. Miller ${ }^{103}$, L. Miramonti ${ }^{46}$, S. Mollerach ${ }^{1}$, M. Monasor ${ }^{98}$, D. Monnier Ragaigne ${ }^{32}$, F. Montanet $^{34}$, B. Morales ${ }^{66}$, C. Morello $^{55}$, E. Moreno ${ }^{61}$, J.C. Moreno ${ }^{6}$, C. Morris ${ }^{94}$, M. Mostafá ${ }^{87}$, S. Mueller ${ }^{37}$, M.A. Muller ${ }^{18}$, G. Müller ${ }^{41}$, M. Münchmeyer ${ }^{33}$, R. Mussa ${ }^{51}$, G. Navarra ${ }^{55}$, J.L. Navarro ${ }^{79}$, S. Navas ${ }^{79}$, P. Necesal ${ }^{27}$, L. Nellen ${ }^{66}$, P.T. Nhung ${ }^{107}$, N. Nierstenhoefer ${ }^{36}$, D. Nitz ${ }^{91}$, D. Nosek ${ }^{26}$, L. Nožka ${ }^{27}$, M. Nyklicek ${ }^{27}$, J. Oehlschläger ${ }^{37}$, A. Olinto ${ }^{98}$, P. Oliva ${ }^{36}$, V.M. Olmos-Gilbaja ${ }^{80}$, M. Ortiz ${ }^{77}$, N. Pacheco ${ }^{78}$, D. Pakk Selmi-Dei ${ }^{18}$, M. Palatka ${ }^{27}$, J. Pallotta ${ }^{3}$, N. Palmieri ${ }^{39}$, G. Parente ${ }^{80}$, E. Parizot $^{31}$, A. Parra $^{80}$, J. Parrisius ${ }^{39}$, R.D. Parsons ${ }^{83}$, S. Pastor ${ }^{76}$, T. Paul ${ }^{93}$, V. Pavlidou ${ }^{98} c$, K. Payet $^{34}$, M. Pech ${ }^{27}$, J. Pȩkala ${ }^{71}$, R. Pelayo ${ }^{80}$, I.M. Pepe ${ }^{21}$, L. Perrone ${ }^{52}$, R. Pesce ${ }^{44}$, E. Petermann ${ }^{102}$, S. Petrera ${ }^{45}$, P. Petrinca ${ }^{49}$, A. Petrolini ${ }^{44}$, Y. Petrov ${ }^{87}$, J. Petrovic $^{69}$, C. Pfendner ${ }^{105}$, N. Phan ${ }^{103}$, R. Piegaia ${ }^{4}$, T. Pierog $^{37}$, P. Pieroni ${ }^{4}$, M. Pimenta ${ }^{73}$, V. Pirronello ${ }^{50}$, M. Platino ${ }^{2}$, V.H. Ponce ${ }^{1}$, M. Pontz ${ }^{43}$, P. Privitera ${ }^{98}$, M. Prouza ${ }^{27}$, E.J. Quel ${ }^{3}$, J. Rautenberg ${ }^{36}$, O. Ravel ${ }^{35}$, D. Ravignani ${ }^{2}$, B. Revenu ${ }^{35}$, J. Ridky ${ }^{27}$, S. Riggi ${ }^{50}$, M. Risse ${ }^{43}$, P. Ristori ${ }^{3}$, H. Rivera ${ }^{46}$, C. Rivière ${ }^{34}$, V. Rizi ${ }^{45}$, C. Robledo ${ }^{61}$, G. Rodriguez ${ }^{80}$, J. Rodriguez Martino ${ }^{9,50}$, J. Rodriguez Rojo ${ }^{9}$, I. Rodriguez$\mathrm{Cabo}^{80}$, M.D. Rodríguez-Frías ${ }^{78}$, G. Ros $^{78}$, J. Rosado ${ }^{77}$, T. Rossler ${ }^{28}$, M. Roth ${ }^{37}$, B. Rouilléd'Orfeuil $^{98}$, E. Roulet ${ }^{1}$, A.C. Rovero ${ }^{7}$, F. Salamida ${ }^{37,45}$, H. Salazar ${ }^{61}$, G. Salina ${ }^{49}$, F. Sánchez ${ }^{2}$, M. Santander ${ }^{9}$, C.E. Santo ${ }^{73}$, E. Santos ${ }^{73}$, E.M. Santos ${ }^{23}$, F. Sarazin ${ }^{86}$, S. Sarkar ${ }^{81}$, R. Sato ${ }^{9}$, N. Scharf ${ }^{41}$, V. Scherini ${ }^{46}$, H. Schieler ${ }^{37}$, P. Schiffer ${ }^{41}$, A. Schmidt ${ }^{38}$, F. Schmidt ${ }^{98}$, T. Schmidt ${ }^{39}$, O. Scholten ${ }^{68}$, H. Schoorlemmer ${ }^{67}$, J. Schovancova ${ }^{27}$, P. Schovánek ${ }^{27}$, F. Schroeder ${ }^{37}$, S. Schulte ${ }^{41}$, F. Schüssler ${ }^{37}$, D. Schuster ${ }^{86}$, S.J. Sciutto ${ }^{6}$, M. Scuderi ${ }^{50}$, A. Segreto ${ }^{54}$, D. Semikoz ${ }^{31}$, M. Settimo ${ }^{47}$, A. Shadkam ${ }^{90}$, R.C. Shellard ${ }^{14,15}$, I. Sidelnik ${ }^{2}$, G. Sigl ${ }^{42}$, A. Śmiałkowski ${ }^{72}$, R. Šmída ${ }^{37,27}$, G.R. Snow ${ }^{102}$, P. Sommers ${ }^{95}$, J. Sorokin ${ }^{11}$, H. Spinka ${ }^{84,89}$, R. Squartini ${ }^{9}$, J. Stapleton ${ }^{94}$, J. Stasielak ${ }^{71}$, M. Stephan ${ }^{41}$, E. Strazzeri ${ }^{54}$, A. Stutz ${ }^{34}$, F. Suarez ${ }^{2}$, T. Suomijärvi ${ }^{30}$, A.D. Supanitsky ${ }^{66}$, T. Šuša ${ }^{25}$, M.S. Sutherland ${ }^{94}$, J. Swain ${ }^{93}$, Z. Szadkowski ${ }^{36,72}$, A. Tamashiro ${ }^{7}$, A. Tapia ${ }^{2}$, T. Tarutina ${ }^{6}$, O. Taşcău $^{36}$, R. Tcaciuc ${ }^{43}$, D. Tcherniakhovski ${ }^{38}$, D. Tegolo $^{50,59}$, N.T. Thao ${ }^{107}$, D. Thomas $^{87}$, J. Tiffenberg ${ }^{4}$, C. Timmermans ${ }^{69,67}$, D.K. Tiwari ${ }^{65}$, W. Tkaczyk ${ }^{72}$, C.J. Todero Peixoto ${ }^{22}$, B. Tomé ${ }^{73}$, A. Tonachini ${ }^{51}$, P. Travnicek ${ }^{27}$, D.B. Tridapalli ${ }^{17}$, G. Tristram $^{31}$, E. Trovato ${ }^{50}$, M. Tueros ${ }^{6}$, R. Ulrich ${ }^{95,37}$, M. Unger ${ }^{37}$, M. Urban ${ }^{32}$, J.F. Valdés Galicia ${ }^{66}$, 
I. Valiño ${ }^{80,37}$, L. Valore ${ }^{48}$, A.M. van den $\mathrm{Berg}^{68}$, B. Vargas Cárdenas ${ }^{66}$, J.R. Vázquez ${ }^{77}$, R.A. Vázquez ${ }^{80}$, D. Veberič ${ }^{75,74}$, T. Venters ${ }^{98}$, V. Verzi ${ }^{49}$, M. Videla ${ }^{8}$, L. Villaseñor ${ }^{65}$, H. Wahlberg ${ }^{6}$, P. Wahrlich ${ }^{11}$, O. Wainberg ${ }^{2}$, D. Warner ${ }^{87}$, A.A. Watson ${ }^{83}$, M. Weber ${ }^{38}$, K. Weidenhaupt ${ }^{41}$, A. Weindl ${ }^{37}$, S. Westerhoff ${ }^{105}$, B.J. Whelan ${ }^{11}$, G. Wieczorek ${ }^{72}$, L. Wiencke ${ }^{86}$, B. Wilczyńska ${ }^{71}$, H. Wilczyński ${ }^{71}$, M. Will ${ }^{37}$, C. Williams ${ }^{98}$, T. Winchen ${ }^{41}$, L. Winders ${ }^{106}$, M.G. Winnick ${ }^{11}$, M. Wommer ${ }^{37}$, B. Wundheiler ${ }^{2}$, T. Yamamoto ${ }^{98} a$, P. Younk ${ }^{87}$, G. Yuan ${ }^{90}$, A. Yushkov ${ }^{48}$, B. Zamorano ${ }^{79}$, E. Zas ${ }^{80}$, D. Zavrtanik ${ }^{75,74}$, M. Zavrtanik ${ }^{74,75}$, I. Zaw ${ }^{92}$, A. Zepeda ${ }^{62}$, M. Ziolkowski ${ }^{43}$

${ }^{1}$ Centro Atómico Bariloche and Instituto Balseiro (CNEA- UNCuyo-CONICET), San Carlos de Bariloche, Argentina

2 Centro Atómico Constituyentes (Comisión Nacional de Energía Atómica/CONICET/UTNFRBA), Buenos Aires, Argentina

${ }^{3}$ Centro de Investigaciones en Láseres y Aplicaciones, CITEFA and CONICET, Argentina

${ }^{4}$ Departamento de Física, FCEyN, Universidad de Buenos Aires y CONICET, Argentina

${ }^{6}$ IFLP, Universidad Nacional de La Plata and CONICET, La Plata, Argentina

${ }^{7}$ Instituto de Astronomía y Física del Espacio (CONICET- UBA), Buenos Aires, Argentina

${ }^{8}$ National Technological University, Faculty Mendoza (CONICET/CNEA), Mendoza, Argentina

${ }^{9}$ Pierre Auger Southern Observatory, Malargüe, Argentina

10 Pierre Auger Southern Observatory and Comisión Nacional de Energía Atómica, Malargüe, Argentina

${ }^{11}$ University of Adelaide, Adelaide, S.A., Australia

${ }^{14}$ Centro Brasileiro de Pesquisas Fisicas, Rio de Janeiro, RJ, Brazil

15 Pontifícia Universidade Católica, Rio de Janeiro, RJ, Brazil

${ }^{16}$ Universidade de São Paulo, Instituto de Física, São Carlos, SP, Brazil

${ }^{17}$ Universidade de São Paulo, Instituto de Física, São Paulo, SP, Brazil

${ }^{18}$ Universidade Estadual de Campinas, IFGW, Campinas, SP, Brazil

${ }^{19}$ Universidade Estadual de Feira de Santana, Brazil

${ }^{20}$ Universidade Estadual do Sudoeste da Bahia, Vitoria da Conquista, BA, Brazil

${ }^{21}$ Universidade Federal da Bahia, Salvador, BA, Brazil

22 Universidade Federal do ABC, Santo André, SP, Brazil

${ }^{23}$ Universidade Federal do Rio de Janeiro, Instituto de Física, Rio de Janeiro, RJ, Brazil

${ }^{25}$ Rudjer Bošković Institute, 10000 Zagreb, Croatia

${ }^{26}$ Charles University, Faculty of Mathematics and Physics, Institute of Particle and Nuclear Physics, Prague, Czech Republic

${ }^{27}$ Institute of Physics of the Academy of Sciences of the Czech Republic, Prague, Czech Republic

${ }^{28}$ Palacký University, Olomouc, Czech Republic

${ }^{30}$ Institut de Physique Nucléaire d'Orsay (IPNO), Université Paris 11, CNRS-IN2P3, Orsay, France

${ }^{31}$ Laboratoire AstroParticule et Cosmologie (APC), Université Paris 7, CNRS-IN2P3, Paris, France

${ }^{32}$ Laboratoire de l'Accélérateur Linéaire (LAL), Université Paris 11, CNRS-IN2P3, Orsay, France

${ }^{33}$ Laboratoire de Physique Nucléaire et de Hautes Energies (LPNHE), Universités Paris 6 et Paris

7, CNRS-IN2P3, Paris, France 
${ }^{34}$ Laboratoire de Physique Subatomique et de Cosmologie (LPSC), Université Joseph Fourier, INPG, CNRS-IN2P3, Grenoble, France

${ }^{35}$ SUBATECH, CNRS-IN2P3, Nantes, France

${ }^{36}$ Bergische Universität Wuppertal, Wuppertal, Germany

37 Karlsruhe Institute of Technology - Campus North - Institut für Kernphysik, Karlsruhe, Germany

${ }^{38}$ Karlsruhe Institute of Technology - Campus North - Institut für Prozessdatenverarbeitung und Elektronik, Karlsruhe, Germany

${ }^{39}$ Karlsruhe Institute of Technology - Campus South - Institut für Experimentelle Kernphysik (IEKP), Karlsruhe, Germany

${ }^{40}$ Max-Planck-Institut für Radioastronomie, Bonn, Germany

${ }^{41}$ RWTH Aachen University, III. Physikalisches Institut A, Aachen, Germany

42 Universität Hamburg, Hamburg, Germany

${ }^{43}$ Universität Siegen, Siegen, Germany

${ }^{44}$ Dipartimento di Fisica dell'Università and INFN, Genova, Italy

${ }^{45}$ Università dell'Aquila and INFN, L'Aquila, Italy

${ }^{46}$ Università di Milano and Sezione INFN, Milan, Italy

${ }^{47}$ Dipartimento di Fisica dell'Università del Salento and Sezione INFN, Lecce, Italy

${ }^{48}$ Università di Napoli "Federico II" and Sezione INFN, Napoli, Italy

${ }^{49}$ Università di Roma II "Tor Vergata" and Sezione INFN, Roma, Italy

${ }^{50}$ Università di Catania and Sezione INFN, Catania, Italy

${ }^{51}$ Università di Torino and Sezione INFN, Torino, Italy

52 Dipartimento di Ingegneria dell'Innovazione dell'Università del Salento and Sezione INFN, Lecce, Italy

${ }^{54}$ Istituto di Astrofisica Spaziale e Fisica Cosmica di Palermo (INAF), Palermo, Italy

${ }^{55}$ Istituto di Fisica dello Spazio Interplanetario (INAF), Università di Torino and Sezione INFN, Torino, Italy

${ }^{56}$ INFN, Laboratori Nazionali del Gran Sasso, Assergi (L'Aquila), Italy

${ }^{59}$ Università di Palermo and Sezione INFN, Catania, Italy

${ }^{61}$ Benemérita Universidad Autónoma de Puebla, Puebla, Mexico

${ }^{62}$ Centro de Investigación y de Estudios Avanzados del IPN (CINVESTAV), México, D.F., Mexico

${ }^{65}$ Universidad Michoacana de San Nicolas de Hidalgo, Morelia, Michoacan, Mexico

${ }^{66}$ Universidad Nacional Autonoma de Mexico, Mexico, D.F., Mexico

${ }^{67}$ IMAPP, Radboud University, Nijmegen, Netherlands

${ }^{68}$ Kernfysisch Versneller Instituut, University of Groningen, Groningen, Netherlands

${ }^{69}$ NIKHEF, Amsterdam, Netherlands

${ }^{70}$ ASTRON, Dwingeloo, Netherlands

${ }^{71}$ Institute of Nuclear Physics PAN, Krakow, Poland

72 University of Łódź, Łódź, Poland

${ }^{73}$ LIP and Instituto Superior Técnico, Lisboa, Portugal

74 J. Stefan Institute, Ljubljana, Slovenia

${ }^{75}$ Laboratory for Astroparticle Physics, University of Nova Gorica, Slovenia

${ }^{76}$ Instituto de Física Corpuscular, CSIC-Universitat de València, Valencia, Spain 
77 Universidad Complutense de Madrid, Madrid, Spain

${ }^{78}$ Universidad de Alcalá, Alcalá de Henares (Madrid), Spain

${ }^{79}$ Universidad de Granada \& C.A.F.P.E., Granada, Spain

${ }^{80}$ Universidad de Santiago de Compostela, Spain

${ }^{81}$ Rudolf Peierls Centre for Theoretical Physics, University of Oxford, Oxford, United Kingdom

${ }^{83}$ School of Physics and Astronomy, University of Leeds, United Kingdom

${ }^{84}$ Argonne National Laboratory, Argonne, IL, U.S.A.

${ }^{85}$ Case Western Reserve University, Cleveland, OH, U.S.A.

${ }^{86}$ Colorado School of Mines, Golden, CO, U.S.A.

${ }^{87}$ Colorado State University, Fort Collins, CO, U.S.A.

${ }^{88}$ Colorado State University, Pueblo, CO, U.S.A.

${ }^{89}$ Fermilab, Batavia, IL, U.S.A.

${ }^{90}$ Louisiana State University, Baton Rouge, LA, U.S.A.

${ }^{91}$ Michigan Technological University, Houghton, MI, U.S.A.

92 New York University, New York, NY, U.S.A.

${ }^{93}$ Northeastern University, Boston, MA, U.S.A.

94 Ohio State University, Columbus, OH, U.S.A.

${ }^{95}$ Pennsylvania State University, University Park, PA, U.S.A.

${ }^{96}$ Southern University, Baton Rouge, LA, U.S.A.

${ }^{97}$ University of California, Los Angeles, CA, U.S.A.

${ }^{98}$ University of Chicago, Enrico Fermi Institute, Chicago, IL, U.S.A.

102 University of Nebraska, Lincoln, NE, U.S.A.

${ }^{103}$ University of New Mexico, Albuquerque, NM, U.S.A.

${ }^{105}$ University of Wisconsin, Madison, WI, U.S.A.

106 University of Wisconsin, Milwaukee, WI, U.S.A.

${ }^{107}$ Institute for Nuclear Science and Technology (INST), Hanoi, Vietnam

$(\dagger)$ Deceased

(a) at Konan University, Kobe, Japan

(c) at Caltech, Pasadena, U.S.A.

\section{References}

[1] K. Herbst et al., On the importance of the local interstellar spectrum for the solar modulation parameter, J. Geophys. Res. 115 (2010) D00I20.

[2] S.E. Forbush, Three Unusual Cosmic-Ray Increases Possibly Due to Charged Particles from the Sun, Phys. Rev. 70 (1946) 771.

[3] H.V. Cane, Coronal Mass Ejections and Forbush Decreases, Space Sci. Rev. 93 (2000) 55.

[4] Pierre Auger collaboration, J. Abraham et al., Properties and performance of the prototype instrument for the Pierre Auger Observatory, Nucl. Instrum. Meth. A 523 (2004) 50.

[5] Pierre Auger collaboration, J. Abraham et al., The Fluorescence Detector of the Pierre Auger Observatory, Nucl. Instrum. Meth. 620 (2010) 227. 
[6] Pierre Auger collaboration, I. Allekotte et al., The Surface Detector System of the Pierre Auger Observatory, Nucl. Instrum. Meth. A 586 (2008) 409.

[7] Pierre Auger collaboration, X. Bertou et al., Calibration of the surface array of the Pierre Auger Observatory, Nucl. Instrum. Meth. A 568 (2006) 839.

[8] Pierre Auger collaboration, P.D.J. Clark and D. Nitz, Communications in the Auger Observatory, in Proceedings of the 27th International Cosmic Ray Conference (ICRC 2001), Hamburg, Germany (2001), pg. 765-768.

[9] P. Bauleo et al., A water tank Cerenkov detector for very high energy astroparticles, Nucl. Instrum. Meth. A 406 (1998) 69.

[10] Pierre Auger collaboration, M. Aglietta et al., Response of the Pierre Auger Observatory Water Cherenkov Detectors to Muons, in Proceedings of the 29th International Cosmic Ray Conference (ICRC 2005), Pune, India (2005), pg. 83-86.

[11] Pierre Auger collaboration, A. Etchegoyen et al., Muon-track studies in a water Cherenkov detector, Nucl. Instrum. Meth. A 545 (2005) 602.

[12] D. Alexander, K.M. Pathak and M.G. Thompson, Cerenkov energy loss of muons in water, J. Phys. A 1 (1968) 578.

[13] C. Morello, L. Periale and G. Navarra, A search for high-energy cosmic gamma-ray bursts, Nuovo Cim. C 7 (1984) 682.

[14] Pierre Auger collaboration, X. Bertou, Search for Gamma Ray Bursts using the single particle technique at the Pierre Auger Observatory, in Proceedings of the 30th International Cosmic Ray Conference (ICRC 2007), Mexico DF, Mexico (2008), pg. 441-444.

[15] D. Allard et al., Use of water-Cherenkov detectors to detect gamma ray bursts at the Large Aperture GRB Observatory (LAGO), Nucl. Instrum. Meth. A 595 (2008) 70.

[16] A. Budano et al., Search for Gamma Ray Bursts with the Argo-YBJ Detector in Scaler Mode, Astrophys. J. 699 (2009) 1281.

[17] Pierre Auger collaboration, D. Thomas, Search for coincidences with astrophysical transients in Pierre Auger Observatory data, in Proceedings of the 31st International Cosmic Ray Conference (ICRC 2009), Lodz, Poland (2009).

[18] ICE CuBE collaboration, R. Abbasi et al., Solar Energetic Particle Spectrum on 2006 December 13 Determined by IceTop, Astrophys. J. $\mathbf{6 8 9}$ (2008) L65.

[19] Pierre Auger collaboration, H. Asorey, Cosmic Ray Solar Modulation Studies at the Pierre Auger Observatory, in Proccedings of the 31 st International Cosmic Ray Conference (ICRC 2009), Lodz, Poland (2009).

[20] E. Eroshenko et al., Relationships between neutron fluxes and rain flows, Adv. Space Res. 46 (2010) 637.

[21] R. Caballero and J.F. Valdés-Galicia, Solar modulation of galactic cosmic-ray intensity as seen by neutron monitors during 1990-1999-I. Rigidity Dependence and Correlations with Solar Activity Parameters, Solar Phys. 212 (2004) 209.

[22] M. Storini et al., Rome Neutron Monitor, supported by INAF/UNIRomaTre collaboration, http://cr0.izmiran.rssi.ru/rome/main.htm.

[23] D.F. Smart and M.A. Shea, Grid of Calculated Cosmic Ray Vertical Cutoff Rigidities for Epoch 2000.0, in Proceedings of the 30th International Cosmic Ray Conference (ICRC 2007), Mexico DF, Mexico (2007), pg. 737-740. 
[24] E.G. Cordaro and E.F. Olivares, Cosmic Rays 6-NM-64 Neutron Monitor Data, Los Cerrillos Cosmic Rays Observatory, in Report of Cosmic Rays Research Laboratory 2005, ISSN 0717-361X, 2005, Santiago, Chile.

[25] J.W. Bieber et al., Spaceship Earth Observations of the Easter 2001 Solar Particle Event, Astrophys. J. Lett. 601 (2004) L103.

[26] T. Kuwabara et al., Determination of interplanetary coronal mass ejection geometry and orientation from ground-based observations of galactic cosmic rays, J. Geophys. Res. 114 (2009) A05109.

[27] T. Mulligan et al., Short-period variability in the galactic cosmic ray intensity: High statistical resolution observations and interpretation around the time of a Forbush decrease in August 2006, J. Geophys. Res. 114 (2009) A07105. 